\title{
Role of protein degradation in growth laws
}

\author{
Ludovico Calabrese $^{1 *}$, Jacopo Grilli ${ }^{2}$, Matteo Osella ${ }^{3}$, Christopher P Kempes ${ }^{4}$, Marco
} Cosentino Lagomarsino ${ }^{1,5,6^{*} \boldsymbol{\otimes}}$, Luca Ciandrini ${ }^{7^{*} \boldsymbol{\ominus}}$,

1 IFOM Foundation, FIRC Institute for Molecular Oncology, via Adamello 16, Milan, Italy

2 The Abdus Salam International Centre for Theoretical Physics (ICTP), Str. Costiera 11, 34151 Trieste, Italy

3 Physics Department, University of Turin and INFN, via P. Giuria 1, 10125 Turin, Italy 4 The Santa Fe Institute, Santa Fe, NM, United States

5 Dipartimento di Fisica, Università degli Studi di Milano, via Celoria 16, Milan, Italy

6 INFN sezione di Milano, via Celoria 16, Milan, Italy

7 Centre de Biologie Structurale (CBS), Univ Montpellier, CNRS, INSERM, Montpellier 34090, France

(2These authors contributed equally to this work.

* For correspondence: ludovico.calabrese@ifom.eu (LCa);

marco.cosentino-lagomarsino@ifom.eu (MCL); luca.ciandrini@umontpellier.fr (LCi)

\begin{abstract}
Growing cells adopt common basic strategies to achieve optimal resource allocation under limited resource availability. Our current understanding of such "growth laws" neglects degradation, assuming that it occurs slowly compared to the cell cycle duration. Here we argue that this assumption cannot hold at slow growth, leading to important consequences. We propose a simple framework showing that at slow growth protein degradation is balanced by a fraction of "maintenance" ribosomes. Consequently, active ribosomes do not drop to zero at vanishing growth, but as growth rate diminishes, an increasing fraction of active ribosomes performs maintenance. Through a detailed analysis of compiled data, we show that the predictions of this model agree with data from $E$. coli and $S$. cerevisiae. Intriguingly, we also find that protein degradation increases at slow growth, which we interpret as a consequence of active waste management and/or recycling. Our results highlight protein turnover as an underrated factor for our understanding of growth laws across kingdoms.
\end{abstract}

\section{Introduction}

"Growth laws" (Scott and Hwa, 2011; Kafri et al., 2016) are quantitative relationships between cell composition and growth rate. They uncover simple underlying physiological design principles that can be used to predict and manipulate cell behavior. One of these laws, sometimes called the "first growth law", relates steady-state growth rate to ribosome allocation, and reflects the fact that the biosynthetic rate is set by the fraction of ribosomes that translate other ribosomes (Scott et al., 2010; Metzl-Raz et al., 2017). Specifically, the mass fraction $\phi_{R}$ of ribosomal proteins in the proteome increases linearly with growth rate $\lambda$, independently of nutrient source. 
Figure 1 provides a visual summary of the relation $\phi_{R}(\lambda)$. Importantly, there is an empirical offset in this law $\phi_{R}(\lambda=0) \neq 0$, i.e., the relationship extrapolates to a nonzero fraction of ribosomes at zero growth. The presence of an offset seems to be widespread across species (Fig. 1 - Supplement 1). This offset is commonly interpreted using the assumption that only a fraction of the total number of ribosomes (sometimes called "active ribosomes") is translating and thus producing mass (Scott et al., 2010, Dai et al. 2016). However, no currently available experimental method is able to quantify active ribosomes, and the origin and nature of the inactive ribosomal pool is under debate (Dai and Zhu, 2020). In E. coli, deviations from this law at slow growth were explained by a growth-rate dependent fraction of active ribosomes (Klumpp et al., 2013, Dai et al. 2016).

Protein degradation and turnover are typically neglected in the frameworks describing growth laws (Scott et al., 2010). Clearly if degradation time scales fall in the range of 10-100 h (Goldberg and Dice, 1974; Maurizi, 1992), they are negligible compared to protein dilution by cell growth when nutrients are abundant. However, when the population doubling time overlaps with the typical time scale of protein degradation, the balance between protein production and protein degradation must impact growth (Maitra and Dill, 2015; Kempes et al., 2016; Santra et al., 2017). Importantly, prolonged slow- or null-growth regimes are of paramount importance in the lifestyle of most bacteria (Kempes et al., 2017; DeLong et al., 2010; Long et al., 2021; Gray et al., 2019; Schink et al., 2019; Biselli et al., 2020), as well as in synthetic biology applications (Borkowski et al., 2016). Notably, the smallest bacterial species not only grow slowly but also have a small number of macromolecules (down to $\approx 40$ ribosomes) suggesting that protein turnover matters in slow growth contexts (Kempes et al., 2016).

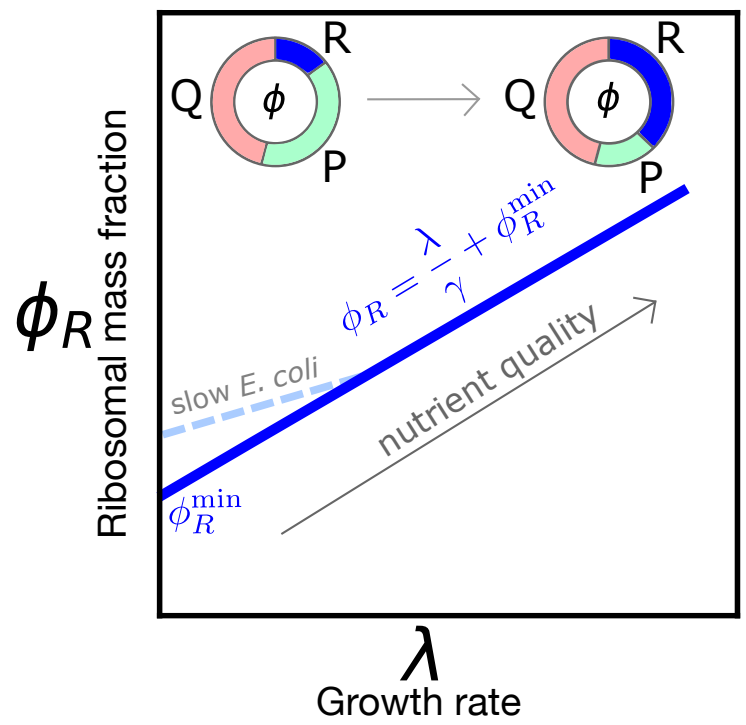

Fig 1. Sketch of the growth law relating ribosome mass fraction $\phi_{R}$ to growth rate $\lambda$. The fraction of ribosomal and ribosome-affiliated proteins $(R)$ increases with increasing nutrient quality at the expense of the sector of metabolic proteins $(\mathrm{P})$, while a fraction of the proteome $(\mathrm{Q})$ is kept to be growth rate-independent. Available data for most organisms show a nonzero intercept $\phi_{R}^{\min }>0$ (see Fig. 1 - Supplement 1). In E. coli (Dai et al., 2016), the law deviates from linearity at slow growth $\left(\lambda \leq 1 \mathrm{~h}^{-1}\right)$, making the intercept $\phi_{R}^{\text {min }}$ larger.

Here, we propose and explore a generic framework to describe the first growth law including the role of protein degradation and turnover (Kempes et al., 2016: Santra et al. 2017). We first derive the law from basic flux-balance principles. We then falsify on 
general grounds a scenario where degradation is not accounted for. Finally, we use our framework on E. coli and $S$. cerevisiae data, finding that data and model converge on a scenario in which, at slow growth, a non-negligible fraction of ribosomes performs maintenance duties, balancing protein degradation, without contributing to growth.

\section{Results}

\section{Degradation sets an offset in the first growth law}

We start by formulating a simple theory for the first growth law that includes degradation. The law can be derived from the following total protein mass $(M)$ flux balance relation, valid for steady exponential growth,

$$
\lambda M=J_{\mathrm{tl}}-J_{\mathrm{deg}} .
$$

Here, $\lambda$ is the cellular growth rate, $J_{\mathrm{tl}}$ is the flux of protein mass synthesized by translation, and we explicitly considered the flux of protein degradation $J_{\mathrm{deg}}$. The term $J_{\mathrm{tl}}$ is proportional to the ribosome current $v \rho$ on a transcript, given by the product between the ribosome speed $v$ and its linear density $\rho$ on an mRNA. This quantity corresponds to the protein synthesis rate if the ribosomal current along a transcript is conserved, i.e. if ribosome drop-off is negligible. We assume that ribosome traffic is negligible, therefore the speed $v$ is independent of $\rho$ and can be identified with the codon elongation rate $k(\operatorname{Li}, 2015)$. In this model, free ribosomal subunits are recruited to mRNAs and become translationally active via a first-order reaction that depends on the concentration of free ribosomes (Fig. 2a).

A simple estimate (see Box 1) shows that $J_{\mathrm{tl}}=m_{\mathrm{aa}} k R$, where $m_{\mathrm{aa}}$ is the typical mass of an amino-acid and $R$ the total number of ribosomes. The flux of protein degradation is determined by the degradation rate $\eta$. We first assume that $\eta$ is a constant that does not depend on the growth rate and it is identical for all proteins, which gives $J_{\operatorname{deg}}=\eta M$. This assumption can be relaxed to study the role of protein-specific degradation rates (see Methods and Materials), but in this work we limit our investigation to the average values of these quantities. Using the expressions for $J_{\mathrm{tl}}$ and $J_{\text {deg }}$ into Eq. (1) and introducing the parameter $\gamma:=k / L_{R}$ (where $L_{R}$ is the number of amino acids in a ribosome), we find a simple relation between the ribosomal protein mass fraction $\phi_{R}$ and the growth rate $\lambda$ that involves the degradation rate,

$$
\lambda=\gamma \phi_{R}-\eta .
$$

Note that $\gamma$ can be interpreted as the inverse of the time needed to translate all the aminoacids needed to build a ribosome. If ribosome speed is growth-rate dependent (Klumpp et al., 2013), $\gamma$ is itself a function of $\lambda$. We will come back to this point in the following.

Equation (2) gives an alternative formulation of the first growth law. Crucially, this equation predicts an offset $\phi_{R}^{\min }=\phi_{R}(\lambda=0)=\eta / \gamma$ in the law, which we can compare to the experimental range of observed offsets, $\phi_{R}^{\min } \sim 0.02-0.1$ (Scott et al., 2010 . Metzl-Raz et al., 2017). Taking $\gamma \approx 3.6-7.2 \mathrm{~h}^{-1}$, this simple estimate returns values for the degradation rate $\eta$ that correspond to a range of (mean) protein half-lives $\sim 1-10$ $\mathrm{h}$. Hence, protein turnover should become relevant for slowly growing cells, when their doubling time falls in the same range of time scales (or is longer). Figure 2 summarizes this result and shows how different degradation rates set different offsets in the predicted linear relationship $\phi_{R}(\lambda)$. In this framework, the offset $\phi_{R}^{\min }=\eta / \gamma$ can be interpreted as the ratio between the time needed for a ribosome to synthesize a new ribosome and the time scale of protein degradation (or decay), which fixes the size of the ribosome 

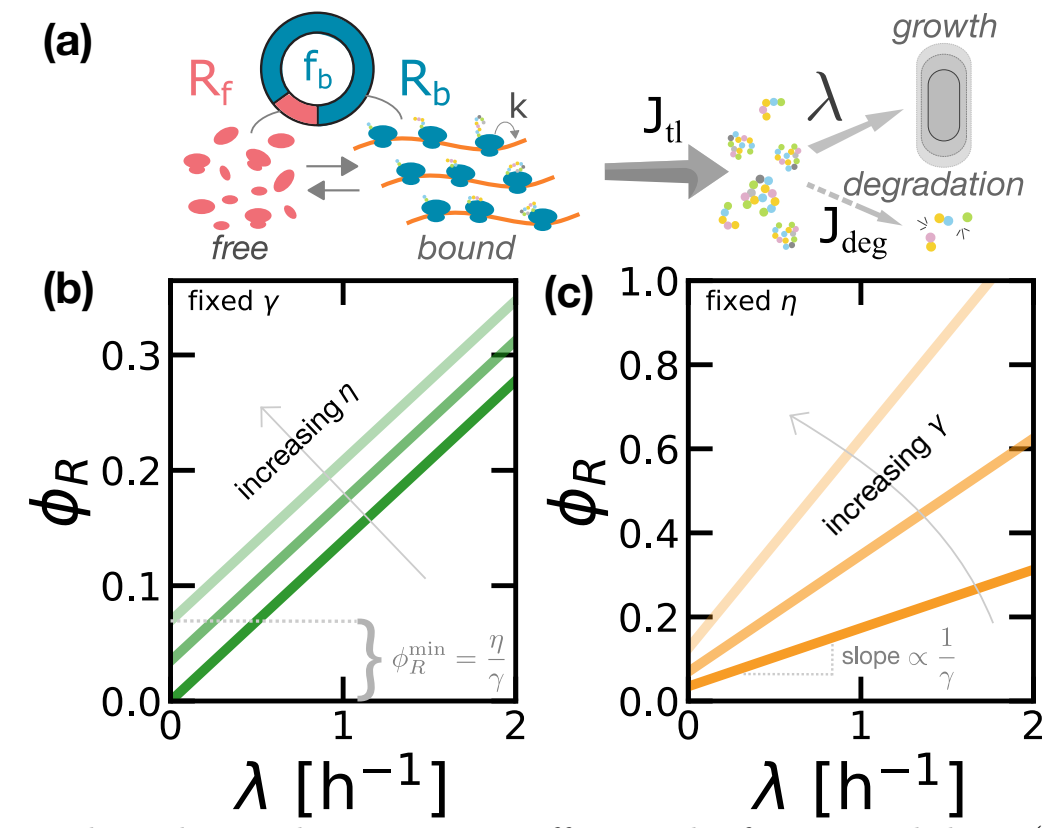

Fig 2. Protein degradation determines an offset in the first growth law. (a) Sketch of the first model of protein production proposed in this work, which includes protein degradation but no inactive ribosomes. In this model, ribosomes follow a first-order kinetics to bind the transcripts, and all bound ribosomes contribute to protein synthesis (mass production). Proteins can be lost by protein degradation or diluted by cell growth. (b) The law $\phi_{R}(\lambda)$ predicted by Eq. (1) shows an offset $\phi_{R}^{\min }=\eta / \gamma$. The offset increases linearly with degradation rate $\eta$ at a constant ribosome production rate $\gamma$. (c) Varying $\gamma$ also changes $\phi_{R}^{\min }$ but it also affects the slope of $\phi_{R}(\lambda)$. Panel (b) reports $\phi_{R}(\lambda)$ for $\gamma=7.2 \mathrm{~h}^{-1}$ and $\eta=0,0.25,0.5 \mathrm{~h}^{-1}$. Panel (c) fixes $\eta=0.25 \mathrm{~h}^{-1}$ and varies $\gamma=2,3.6,7.2 \mathrm{~h}^{-1}$.

pool in steady growth. In other words, the offset $\phi_{R}^{\min }$ can be interpreted as the mass fraction of "maintenance ribosomes", which are needed to sustain protein synthesis in resource-limited conditions.

\section{Box 1. The first growth law in the degradation model.}

At steady growth, mass balance imposes that the fluxes of mass production $J_{\mathrm{tl}}$ and degradation $J_{\text {deg }}$ should be equal

$$
\frac{d M}{d t}=\lambda M=J_{\mathrm{tl}}-J_{\mathrm{deg}} .
$$

The biosynthesis flux is proportional to $j_{m}$, the overall translation rate of the typical transcript, $J_{\mathrm{tl}}=m_{p} N_{m} j_{m}$, where $m_{p}$ is the mass of the typical protein, and $N_{m}$ is the number of transcripts. Assuming a small translation initiation rate, and thus a low ribosome density on each transcript (Ciandrini et al., 2013), the overall translation rate is $k \rho$, and following (Shaw et al., 2003) the density of ribosomes is

$$
\rho=\frac{\frac{\alpha}{k}}{1+(\ell-1) \frac{\alpha}{k}}
$$

where $\ell$ is the size of the ribosome in units of codons (i.e. $\ell \approx 10$ ) and $\alpha$ is the translation initiation rate. Since initiation is about two order of magnitudes slower 
compared to elongation, (0.1 vs $\left.10 \mathrm{~s}^{-1}\right)$ (Ciandrini et al., 2013), the density can be approximated as $\rho \approx \alpha / k$. Describing initiation as a first-order chemical reaction, $\alpha=\alpha_{0} c_{f}$, with $c_{f}$ being the concentration of free ribosomes in solution. Considering that the total number of ribosomes is given by $R=R_{b}+R_{f}$, we obtain the following relation between $R_{f}$ and $R$ (Greulich et al., 2012)

$$
R_{f}=\frac{k R}{k+L c_{m} \alpha_{0}}
$$

where we have introduced the concentration of transcript $c_{m}$. In this theory, the quantity $f_{b}=R_{b} / R$ describes the fraction of bound and translating ribosomes. If the total expected time to elongate a typical protein $\tau_{e}=L / k$ is large compared the time that a ribosome remains unused in the cytoplasm $\tau_{i}=1 / \alpha_{0} c_{m}$, then $j_{m} \simeq k \rho=\alpha_{0} c_{f} \simeq k R /\left(L N_{m}\right)$, and the mass production term reads

$$
J_{\mathrm{tl}}=m_{\mathrm{aa}} k R .
$$

The contribution of protein turnover to the mass balance is $J_{\operatorname{deg}}=\eta M$. Thus, by using the relations for $J_{\mathrm{tl}}$ and $J_{\mathrm{deg}}$ in Eq. (1) we obtain $\lambda=\gamma \phi_{R}-\eta$ - Eq. (2) in the text. We remind that $\phi_{R}=M_{R} / M$ where $M_{R}=m_{R} R$ is the total mass of ribosomal proteins and $m_{R}$ the protein mass of a single ribosome, and that $\gamma=k / L_{R}$ where $L_{R}=m_{R} / m_{\text {aa }}$ is the number of amino acids in a ribosome. The quantity $\gamma^{-1}$ can hence be interpreted as the typical time needed for a ribosome to duplicate its protein content.

\section{The standard framework for the first growth law neglects protein turnover}

To illustrate how the framework involving protein degradation provides a complementary interpretation of the players generating the first growth law, we now discuss the more standard derivation of the relationship $\phi_{R}(\lambda)$, within the usual model where the degradation rate is set to zero. The standard framework neglects protein turnover in all regimes and assumes that only a fraction $f_{a}$ of ribosomes actively translates the transcriptome, while the remaining subset of ribosomes does not contribute to protein synthesis (Figure 2 - Supplement 1 1 ). Thus, among the total number $R$ of ribosomes, $R_{i}$ are considered as inactive, and only $R_{a}=f_{a} R$ active ribosomes elongate the newly synthesized proteins with rate $k$ per codon and generating a mass flux $J_{\mathrm{tl}}$. It is yet experimentally unfeasible to distinguish between active and inactive ribosomes $(\mathrm{Zhu}$ et al. 2020), and growth laws are typically formulated in terms of the total ribosome to total protein mass fraction $\phi_{R}$. After a few rearrangements (see Box 2), we write

$$
\lambda=\gamma\left(\phi_{R}-\phi_{R}^{i}\right)=\gamma \phi_{R} f_{a},
$$

where $\phi_{R}^{i}$ is the mass protein fraction of inactive ribosomes and $f_{a}=\left(1-R_{i} / R\right)$ is the fraction of actively translating ribosomes, which is in principle a function of the growth state $\lambda$. Note that all inactive ribosomes are considered as they were sequestered in this model, differently from the pool of cytoplasmic ribosomes introduced in the previous section, which are not translating but follow an equilibrium binding kinetics with transcripts as shown in Fig. 2a.

The active ribosomes framework predicts an offset in the linear relation $\phi_{R}(\lambda)$, which originates from the fraction of inactive ribosomes $\phi_{R}^{i}$ at zero growth. When mass is not produced $(\lambda=0)$, in this model there are no ribosomes that are actively translating proteins, but there exists a non-vanishing fraction of inactive ribosomes. Note that Eq.(2) from the "degradation" model, and Eq.(7) from the "active ribosomes" model are 
mathematically equivalent if we identify the degradation rate $\eta$ in the first model with the product $\gamma \phi_{R}^{i}$ in the second. Hence, the two frameworks give a different interpretation of the mechanisms generating the offset in the ribosomal fraction at vanishing growth.

After detailing in the next sections why inactive ribosomes alone cannot explain the offset $\phi_{R}^{\min }$ and gathering available data on protein degradation rates, we will propose a second model including both protein degradation and inactive ribosomes.

\section{Box 2. Active ribosomes model}

Assuming balanced exponential growth, all cellular components accumulate at the same rate $\lambda$. Neglecting protein turnover, the exponential increase of the total protein mass $M$ is

$$
\frac{d M}{d t}=\lambda M
$$

The mass production term is usually expressed as the product between the number of actively translating ribosomes $R_{a}$, the codon elongation rate $k$ and the mass of an amino acid $m_{a a}$ (Dai et al., 2016):

$$
\frac{d M}{d t}=m_{\mathrm{aa}} k R_{a}
$$

Equations (8) and (9) lead to a relation between the growth rate $\lambda$ and the mass fraction of $R_{a}$. However, the number of actively translating ribosomes $R_{a}$ is not easily accessible experimentally. Instead, one can express it in terms of the total number of ribosomes, $R=R_{i}+R_{a}$, where $R_{i}$ is the number of inactive ribosomes. This gives

$$
\lambda=\gamma\left(\phi_{R}-\phi_{R}^{i}\right)=\gamma \phi_{R} f_{a}(\lambda),
$$

which gives an offset in the first growth law, related to the fraction of active ribosomes $f_{a}(\lambda)$.

\section{Analysis of the slow-growth regime supports a scenario where protein degradation cannot be neglected}

We will now argue on general grounds that if protein turnover is not included in a description of growth laws of slowly-growing cells, the description becomes inconsistent.

To this aim, we compare more closely the degradation model with the standard approach. The active ribosomes model (Box 2 and Fig. 2 - Supplement 1 ) predicts that the fraction of active ribosomes $f_{a}$ is always less than 1 and it adapts to the growth state. Assuming that the fraction of active ribosomes $f_{a}$ is also a function of $\lambda$, one obtains the relationship

$$
f_{a}(\lambda)=\frac{\lambda}{\gamma(\lambda) \phi_{R}(\lambda)} .
$$

Since $\phi_{R}(\lambda)$ must be finite for vanishing growth rates, Eq. (11) implies that the fraction of active ribosomes must disappear, unless the protein synthesis rate $\gamma(\lambda)$ falls linearly to zero. In the case of $E$. coli, for example, the measured elongation rate $k$ is different from zero at vanishing growth rate (Dai et al., 2016); given the observed nonzero $\phi_{R}^{\min }$, this theory would predict the complete absence of active ribosomes (Figure 2 - Supplement 1), in contrast with the experimental measurement of a finite translation elongation rate. Conversely, it is reasonable to expect that for maintenance purposes, a subset of ribosomes would remain active and the translation elongation rate $\gamma$ could be nonzero for growth rates comparable to the time scales of protein degradation. We also note that in bacteria 
maintenance protein synthesis is reported to be active even in stationary phase (Gefen et al., 2014). These considerations suggest that, while combined scenarios are possible (see below), and inactive ribosomes can also play a role, protein turnover should not be neglected in a theoretical description of the determinants of the first growth law and the origin of the offset $\phi_{R}^{\min }$.

\section{Compiled degradation-rate data show a tendency of degradation rates to increase with decreasing growth rate.}

Given the differences with the standard framework, we proceeded to test the degradation model more stringently with data. We compiled data from the literature relative to degradation rates in E. coli and S. cerevisiae. These data are available as a Mendeley Data repository (see Methods and Materials).

Despite of the burst of recent quantitative experiments connected to the discovery of growth laws, there are no recent systematic and quantitative measurements of protein degradation in E. coli, but many such measurements are available from classic studies (Goldberg and Dice, 1974; Maurizi, 1992; Pine, 1970; Nath and Koch, 1971; Pine, 1973; Mosteller et al., 1980; Larrabee et al., 1980; Schroer and St. John, 1981), some of them are reported in Figure 2 - Supplement 2 and 2 - Supplement 3. The most comprehensive summary is found in ref. (Pine, 1973), therefore we mined these data for average degradation rates (there are variations in protein-specific degradation rates (Mosteller et al., 1980; Larrabee et al., 1980; Schroer and St. John, 1981), which we did not consider here). Data from yeast are reported in Figure 2 - Supplement 4 Looking at these data, we noticed a general tendency for mean degradation rates to increase with decreasing growth rate.

\section{A combined model accounting for both active ribosomes and protein turnover predicts that protein degradation always increases the fraction of active ribosomes.}

The simple setting of the degradation model shown in Figure 2 assumed that the degradation rate were independent of the growth rate and that inactive ribosomes were not present. We consider now an extended framework including two additional ingredients. First, as mentioned above, the data show that $\eta$ can be a function of the growth state, but this extension of the model is fairly straightforward, as it amounts to treating this parameter as a function of $\lambda$. Second, this extended framework also jointly includes inactive ribosomes, defined as idle ribosomes that do not contribute to the pool of free ribosomes.

To understand this joint model, we can repeat the procedure followed for the degradation model, splitting the unbound ribosome pool into free and inactive fractions, as sketched in Fig. 3a. Only free ribosomes can bind mRNA (and thus become translationally active). The growth law can be written as

$$
\lambda=\gamma\left(\phi_{R}-\phi_{R}^{i}\right)-\eta(\lambda)=\gamma \phi_{R} f_{b}-\eta(\lambda),
$$

where both the fraction $f_{b}$ of bound/active ribosomes and the role of growth-dependent protein turnover are taken into account. Note that in this notation the fraction $f_{b}$ of bound/active ribosomes ("bound" and "active" can be regarded as synonyms for this model) corresponds to the fraction of active ribosomes $f_{a}$ used in the standard model without degradation (which assumes that all active ribosomes are bound). Indeed, $f_{a}=f_{b}(\eta=0)$, and we present a more detailed comparison below. 
In the combined model, increasing values of the protein degradation rate always increment the estimates for the active ribosome fraction for a given growth rate and for the total ribosome fraction. This is a direct consequence of Eq. 12 , since $f_{b}=\frac{\lambda}{\gamma \phi_{R}}+\frac{\eta}{\gamma \phi_{R}}$. This equation implies the inequality

$$
\frac{\lambda}{\gamma \phi_{R}} \leq f_{b} \leq 1
$$

which defines a lower bound (in absence of degradation) and an upper bound (when all ribosomes are active, including those that do not perform net biosynthesis and perform maintenance) for the bound/active ribosome fraction at any given growth rate. Below the lower bound, too few ribosomes are active to sustain a given level of growth, even in
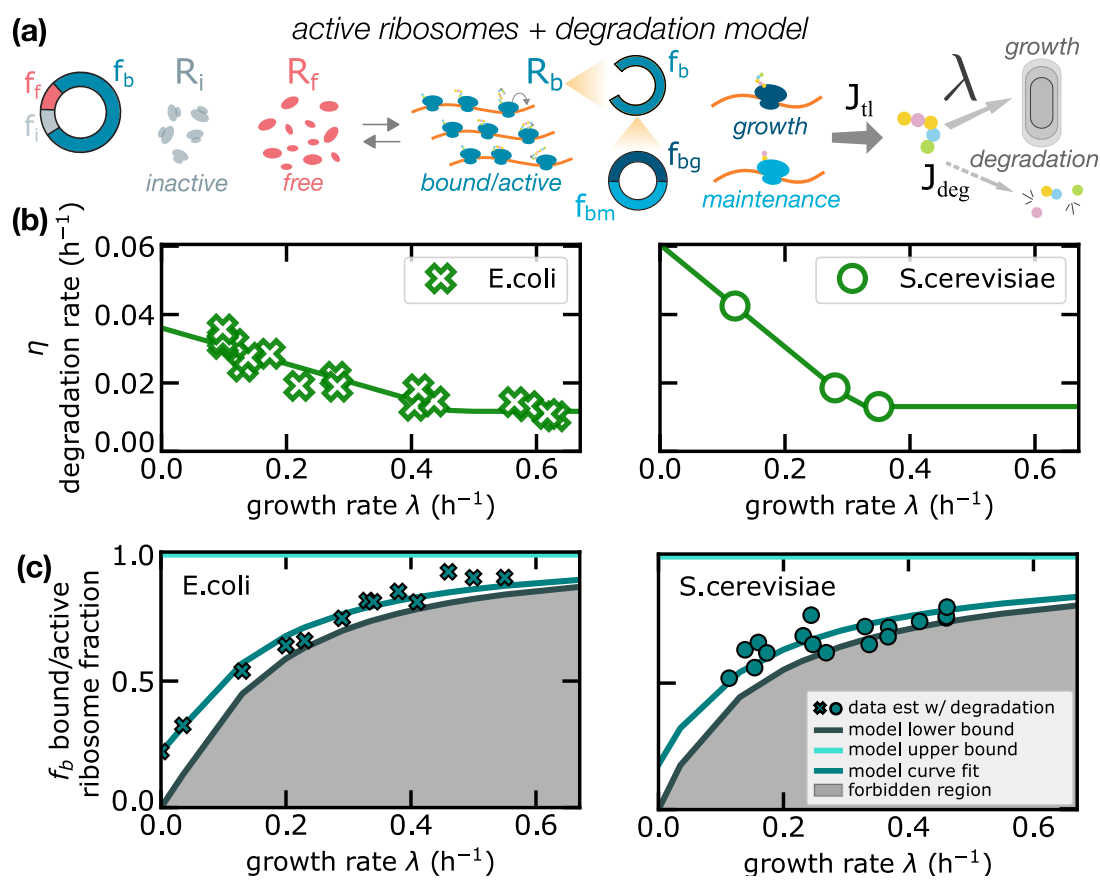

Fig 3. Protein degradation increases the fraction of active ribosomes. (a) Sketch of the second model of protein production proposed in this work, which includes both protein degradation and inactive ribosomes. In this model, only some ribosomes contribute to protein synthesis. As the model in figure 2, proteins can be lost by protein degradation or diluted by cell growth. (b) Experimental data on mean degradation rates across conditions for E. coli from (Pine, 1973) and for S. cerevisiae from (J M Gancedo, 1982). (c) Estimated fraction of active ribosomes in the combined model (turquoise symbols) compared to the model neglecting degradation rates (white symbols). In absence of degradation, the fraction of active ribosomes is estimated from Eq. (7), $f_{b}=\frac{\lambda}{\gamma \phi_{R}}$. In presence of degradation, Eq. 12 gives $f_{b}=\frac{\lambda}{\gamma \phi_{R}}+\frac{\eta}{\gamma \phi_{R}}$. Turquoise symbols (crosses for $E$. coli, circles for $S$. cerevisiae) show the estimates from these formulas for experimental values of the other parameters. The model lower bound (solid line above the shaded area) for the fraction of active ribosomes is the prediction of the active ribosome model, but incorporating the non-null measured degradation rates in these estimates determines considerable deviations from this bound, validating the joint model. Continuous lines are analytical predictions with the constant ratio ansatz, Eq. (14). For E. coli, estimates are performed using data for ribosome fractions $\phi_{R}$ and translation rate $\gamma$ from (Dai et al. 2016) and degradation rates $\eta$ from (Pine, 1973). For S. cerevisiae, estimates are performed using data for ribosome fractions and translation rate from (Metzl-Raz et al. 2017 ) and degradation rates from (J M Gancedo, 1982). 
the absence of degradation. Within this region of existence, the bound/active ribosome fraction depends on the growth rate, following Eq. 12.

Figure 3 c shows the bound/active ribosome fraction estimated by the joint model, using our compiled data for both E. coli and S. cerevisiae (shown in panel b of the same figure). When taking into account the measured degradation rates, in both cases the data confirm the better performance of the combined model (turquoise) with respect to the standard framework that neglects protein turnover (set by the lower bound enclosed in the shaded area). We found that the data agree well with the analytical ansatz

$$
f_{b}(\lambda)=f_{b 0}+\left(1-f_{b 0}\right) \frac{\lambda}{\gamma \phi_{R}}
$$

where $f_{b 0}$ is a constant corresponding to the fraction of active ribosomes at null growth, where all active ribosomes perform maintenance. The constant-ratio ansatz can be validated more directly with data. Indeed, combining Eq. (14) with Eq. [12, we find the linear relationship

$$
\frac{\eta}{\gamma \phi_{R}}=f_{b 0}\left(1-\frac{\lambda}{\gamma \phi_{R}}\right),
$$

which can be verified with a scatter plot of $\eta /\left(\gamma \phi_{R}\right)$ versus $1-\lambda /\left(\gamma \phi_{R}\right)$. This plot, shown in Figure 3 - Supplement 1, shows that the data cluster around a line with constant slope, which estimates $f_{b 0}$. Interestingly, we find that $f_{b 0} \simeq 0.2$ for both $E$. coli and S. cerevisiae data.

This model also confirms the need of including the presence of inactive ribosomes to explain the data. In the Methods and Materials we show that a model without inactive ribosomes (corresponding to $f_{b}=f_{b 0}=1$ ), while capturing the decreasing trend of the degradation rate with the growth rate, is not quantitatively consistent with the data at slow growth (see Fig. 2 - Supplement 3). Additionally, we find that the ansatz of Eq. (14) is equivalent to stating that the fraction of inactive ribosomes is proportional to maintenance ribosomes across growth conditions, therefore we decided to term it "constant ratio" (see Methods and Materials). The constant-ratio ansatz defines a one-parameter family of curves, where the only parameter is the fraction of active ribosomes at null growth $f_{b 0}$, which captures the trend of the active ribosome fractions with the growth for different growth rates and levels of protein degradation (shown in Figure 3b). From those relations one obtains a set of curves $\eta(\lambda)$ showing how the quantitative relation between growth and degradation rates depends on the parameter $f_{b 0}$ (Figure 3 - Supplement 2).

\section{The fraction of active ribosomes increases with protein degradation due to the added presence of ribosome devoted to maintenance}

Taken together, the above analyses favour a scenario of biosynthesis where both degradation and inactive ribosomes cannot be neglected in a wide range of growth rates. We now proceed to quantify more precisely the maintenance component in the combined model, i.e. the balance between protein production and degradation, in this model. To this end, we split active ribosomes into two sub-categories: "growth" bound/active ribosomes, whose fraction is $f_{b g}$, and "maintenance" bound/active ribosomes, whose fraction is $f_{b m}$. The former represents the ribosomes whose protein production contributes to cellular growth, while the latter corresponds to the actively translating ribosomes balancing protein degradation. The two fractions can be defined from the following equations,

$$
\phi_{R}^{b m}:=f_{b m} f_{b} \phi_{R}=\frac{\eta}{\gamma} ; \quad \phi_{R}^{b g}:=f_{b g} f_{b} \phi_{R}=\frac{\lambda}{\gamma},
$$


with $f_{b m}+f_{b g}=1$. Taking the ratio of these expressions, we obtain that such quantities depend only on the ratio $\frac{\eta}{\lambda}$

$$
f_{b g}=\frac{1}{1+\frac{\eta}{\lambda}} ; \quad f_{b m}=\frac{\frac{\eta}{\lambda}}{1+\frac{\eta}{\lambda}} .
$$

Equations (17) have a simple interpretation. Without degradation, all ribosomes contribute directly to growth. Conversely, a fraction of ribosomes needs to be allocated to re-translate the amino acids from degraded proteins

Figure 4 a compares the model predictions for the maintenance ribosome fraction $f_{b m}$ as a function of the protein degradation rate and for fixed values of the growth rate $\lambda$. The combined model predicts that the share of active ribosomes committed to maintenance grows with degradation rate $\eta$, with a trend that depends on the growth rate $\lambda$. Equations (17), clearly show that the different curves collapse when plotted as a function of $\eta / \lambda$ (see inset). To illustrate how these predictions relate to data, we first need to infer the total fraction of bound/active ribosomes. This can be done starting from the fraction of active ribosomes $f_{a}$, as previously defined for the model without degradation in Eq. (11). Comparing with Eq. (12), it is straightforward to relate $f_{b}$ and $f_{a}$, by

$$
f_{b}=f_{a} \frac{\lambda+\eta}{\lambda},
$$

and the fraction of maintenance ribosomes $f_{b m}$ can be computed from Eq.(16) as

$$
f_{b m}=\frac{\eta}{\gamma f_{b} \phi_{R}} .
$$

(a)

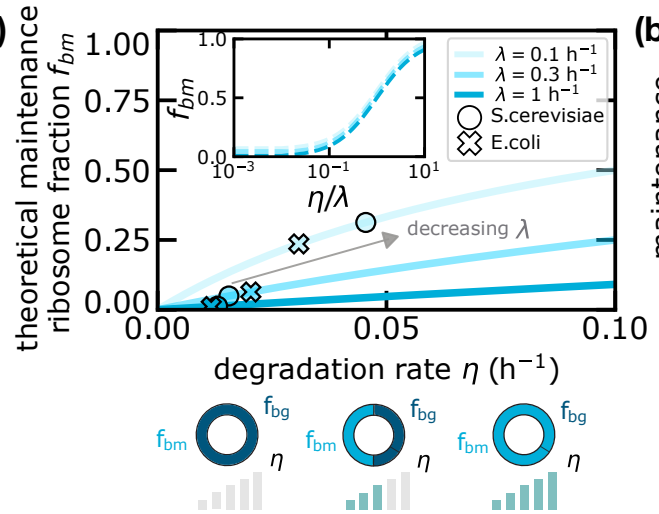

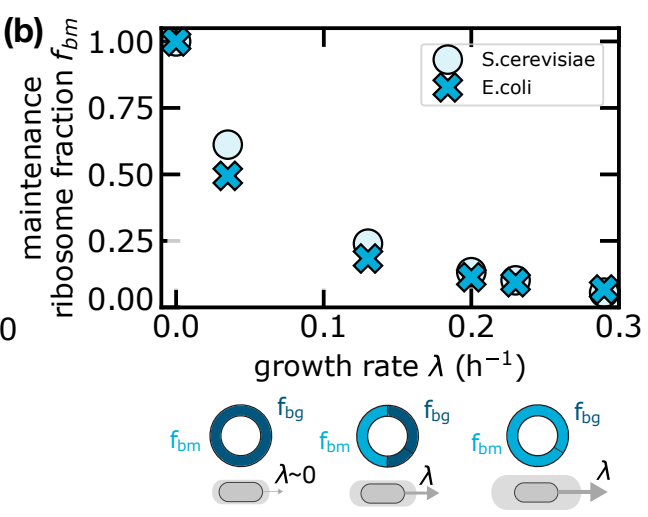

Fig 4. Maintenance ribosomes are responsible for the increase in active ribosomes in the presence of degradation. (a) Theoretical curves (in the combined model) of the maintenance ribosome fraction as a function of the degradation rate $\eta$ for different fixed growth rates $\lambda$. Crosses and circles are obtained from experimental data in $E$. coli and $S$. cerevisiae respectively. Since $f_{b m}$ is a function of the ratio $\eta / \lambda$ only, the inset shows that such curves collapse if plotted as a function of the degradation-to-growth rate ratio. (b) Maintenance ribosome fraction as a function of growth rate estimated from data, for $S$. cerevisiae and E. coli. The fraction of maintenance ribosomes is mathematically identical to the relative difference in total active ribosome fraction between the degradation-only model and the standard framework without degradation. Equivalently, the fraction of active ribosome increases in the presence of degradation due to maintenance ribosomes. Degradation data were derived from (Pine, 1973) (E. coli) and (J M Gancedo, 1982) (S. cerevisiae). Total ribosome fraction data used in Eq. 19) to estimate $f_{b m}$ come from (Dai et al., 2016) (E. coli) and (Metzl-Raz et al., 2017) (S. cerevisiae). 
Fig. 4 a shows how the fractions of maintenance ribosomes derived from experimental data in $S$. cerevisiae and $E$. coli quantitatively lie in the theoretical prediction.

Interestingly, the fraction of active ribosomes devoted to maintenance $f_{b m}$ as given in Eq. (19) also corresponds to the relative difference $\left(f_{b}-f_{a}\right) / f_{b}$ between the predicted fractions of active ribosomes in the models with and without degradation. Therefore, such observable is crucial to understand the effect of degradation on the fraction of active ribosomes. We plot this quantity in Fig. 4 b, showing that at slow growth a non-negligible fraction of ribosomes remains active and, approaching the null-growth state, the vast majority of active ribosomes performs maintenance. Figure $4 \mathrm{~b}$ shows that the fractions of maintenance ribosomes estimated from experimental data are very similar in $E$. coli and $S$. cerevisiae, confirming the idea that this quantity might be dictated by general mass-balance requirements (which is also in line with the fact that the constant-ratio ansatz is verified in the data with similar ratio for the two organisms).

\section{Discussion and Conclusions}

The concepts of maintenance and turnover are central in biosynthesis, and become particularly relevant for slow-growing cells. It seems natural that they would play a role in growth laws. While some recent studies on $E$. coli have focused on biomass recycling from dead cells (Schink et al., 2019; Biselli et al., 2020), here we provide a complementary interpretation for the determinants of the "first growth law" relating ribosome fraction to growth rate in different nutrient conditions. The idea that protein degradation would make the relationship between ribosomal sector and growth rate linear but not proportional was first suggested by Bosdriesz et al. (2015), but this study only commented briefly on this possibility, and did not explore its implications. The concepts introduced here clarify some important aspects on the behavior of slowly-growing $E$. coli. Specifically the relative fraction of inactive ribosomes must be smaller than previously expected, in particular at vanishing growth. In this regime, data and models converge on a scenario where protein degradation sets an increasing set of maintenance ribosomes, which become all active ribosomes at vanishing growth. Thus, in contrast with the widespread notion that at slow growth the fraction of active ribosome tends to disappear, we suggest that ribosome turnover determines a reservoir of active ribosomes at vanishing growth. A further question highlighted by our analysis concerns the causes and the mechanistic determinants of the increase in degradation rates observed at slow growth. While classic studies have observed this effect (Goldberg and Dice, 1974, Maurizi, 1992, Pine, 1973), there are several candidate biological mechanisms underlying this change. Misfolding and protein aggregation occur when translation is slow (Maurizi, 1992), and one could speculate that enhanced protein degradation contributes to the removal of waste products. Other hypotheses see protein degradation as a strategy to strengthen the recycling of amino acids under limited-nutrient conditions, or as a post-translational control mechanism that would tune the levels of specific proteins (Goldberg and Dice, 1974; Maurizi, 1992; Pine, 1973). We also remark that the observed increase of the average degradation rate may also result from the variability of the protein mass fractions in different growth regimes. Here, we did not consider protein-specific degradation rates. However, we can establish a minimal framework with degradation rates $\eta_{R}$ and $\eta_{P}$ that are specific to two corresponding protein sectors $\phi_{R}$ and $\phi_{P}$ (typically representing a ribosomal and a metabolic sector). Equation (2) still holds redefining $\eta$ as

$$
\eta:=\eta_{R} \phi_{R}+\eta_{P} \phi_{P}=\eta_{P}\left(1-e \phi_{R}\right)
$$


i.e. as the weighted average of the degradation rates of the corresponding sectors, with $e:=1-\eta_{R} / \eta_{P}$ and assuming $\phi_{R}+\phi_{P}=1$ for simplicity. Equation 20 indicates that the growth-dependence of $\eta$ might also emerge from the variability of the mass fractions $\phi$ at different physiological states. Unfortunately no experimental data currently allow us to validate this scenario, hence we stuck to the most parsimonious assumption of a common rate. However, we do note that interspecific predictions of the ribosome abundance based on protein abundance and growth rate use this modification and can describe data for diverse species (Kempes et al., 2016). This connection highlights the importance of future work that considers the interplay of shifts in protein abundance, degradation rates, and transcript partitioning across species. Selective degradation of nonribosomal proteins under slow growth has been proposed to play an important role in determining optimal energy efficiency in slow-growing bacteria (Maitra and Dill, 2015).

Beyond E. coli, we expect that the concepts developed here should be even more important for our understanding of growth laws in slow-growing bacteria and eukaryotes. In yeast, protein turnover has been quantified precisely (Christiano et al., 2014), and protein-specific and regulatory aspects of protein degradation and turnover are well known. In particular, selective degradation rates for ribosomal and different kinds of metabolic proteins in different regimes have been reported (Martin-Perez and Villén, 2017; Christiano et al., 2014, Belle et al., 2006; Helbig et al., 2011), which should affect the first growth law (Metzl-Raz et al., 2017). Finally, eukaryotic cells have been reported to activate the expression of autophagy proteins at slow growth, also targeting ribosomes (Beese et al. 2020). However, these aspects remain unexplored from the quantitative standpoint. We expect protein turnover to be relevant in other eukaryotic cells, as post-translational control becomes more common in setting protein concentrations; for instance, fibroblasts increase degradation rates of long-lived proteins as they transition from a proliferating to a quiescent state (Zhang et al., 2017).

We have shown that protein degradation should be taken into account to provide more accurate estimates of the fraction of actively translating ribosomes. Importantly, expressing the ribosomal fraction $\phi_{R}=\Lambda / f_{b}$ as a function of the dimensionless parameter $\Lambda:=(\lambda+\eta) / \gamma$ restores the linearity of the first growth law. This fact highlights the relevance of the relative role of the time scales of ribosome production $(\gamma)$ and dilution/degradation $(\lambda+\eta)$ in determining the fractional size of the ribosomal sector $\phi_{R}$. To test these ideas more stringently, an ideal experimental setup would be capable of informing on ribosomal mass fraction, protein degradation and elongation rate for a wide range of growth rates. This would make it possible to quantify the bound/active ribosome fraction $f_{b}$. Indeed, deviations from linearity in $\phi_{R}(\Lambda)$ would indicate a growth dependence of the fraction of bound/active ribosomes.

In conclusion, our results lead us to conclude that protein turnover is needed to explain important features of cellular resource allocation underlying the growth laws, in particular at slow growth, when the time scales of mass loss for protein degradation and dilution become comparable. In such conditions, differential degradation of proteins with different functions and expression levels will likely play a role in determining physiological responses that yet escape our knowledge. A new generation of large-scale studies of protein-specific degradation, starting from E. coli, may help us building a condensed and quantitative picture of global cell physiology that includes protein turnover. 


\section{Methods and Materials}

\section{Models}

We discuss three different models throughout this study. The "degradation model" (Box 1) provides the relation $\phi_{R}(\lambda)$ by considering the contribution of protein degradation - Eq.(2). The "active ribosome" model, leading to Eq.(7), is our formulation of the standard theory that neglects protein turnover (Dai et al., 2016) (Box 2). The third model that we develop in the last section comprises both aspects of the previous theories (protein degradation and existence of a pool of inactive ribosomes) and is obtained by the procedure explained in Box 1 and considering a total number of ribosomes $R=R_{f}-R_{i}-R_{b}$. Thus, Eq.(5) becomes $R_{f}=k\left(R-R_{i}\right) /\left(k+L c_{m} \alpha_{0}\right)$ and, upon the same hypotheses explained in Box 1, it leads to Eq. (12).

\section{Pure degradation model and data}

This paragraph discusses the comparison of the degradation model (which neglects the presence of inactive ribosomes) with data. This model corresponds to the case $f_{b}=f_{b 0}=1$ in Eq. (12). Under this assumption, the dependency of the degradation rate on the growth rate can be predicted from the data Specifically, assuming the degradation model -Equation (2)- and using the data from Dai and coworkers, we derived the following prediction for the growth-rate dependent degradation rate $\eta$,

$$
\eta(\lambda)=\gamma(\lambda) \phi_{R}(\lambda)-\lambda
$$

The estimated degradation rate, assuming this model, is plotted in Fig. 2 - Supplement 3 The model prediction captures the growth-rate dependence of protein degradation rates observed experimentally, suggesting that deviations from linearity in $\phi_{R}(\lambda)$ could originate at least in part from the increase of degradation rate $\eta$ at slow growth. However, measured values for $\eta$ (Fig. 2 - Supplement 3b) are about one fifth of the model predictions (Fig. 2 - Supplement 3k), indicating that the degradation model alone cannot explain the experimental data, and the inactive ribosomes present in the standard theory, also play a role, as considered in the full model in Eq. (12).

We further tested the degradation model in $S$. cerevisiae, where ribosome allocation data appear to be compatible with the predictions of the model, but this may be due to uncertainty in the data. The available data on yeast do not allow a stringent analysis comparable to the one we could perform for E. coli. Data for ribosome allocation at slow growth rates is lacking (Metzl-Raz et al., 2017), and precise measurements of translation rates -comparable to the analysis of Dai et al. (2016) are not available. Additionally, degradation data across growth conditions are not abundant. However, by taking degradation rate data from J M Gancedo (1982), and a range of translation rates from Boehlke and Friesen (1975) it was possible for us to show that the observed data for the first growth law are in line with the prediction of the model. The results of this analysis (Fig. 2 - Supplement 4) suggest that in this case degradation may fully explain the offset of the first growth law, but more precise experimental data would be needed to establish this point.

\section{Constant-ratio ansatz}

This paragraph further illustrates the meaning of the constant-ratio ansatz introduced in the joint model Eq. (14), which implies that the ratio between inactive ribosomes and ribosomes devoted to maintenance is constant. After multiplying Eq. (15) by $\phi_{R}$ and 
using definition 16 we obtain $\phi_{R}^{b m}=f_{b 0}\left(\phi_{R}-\phi_{R}^{b g}\right)$ or equivalently $\phi_{R}^{b m}=f_{b 0}\left(\phi_{R}^{i}+\phi_{R}^{b m}\right)$, where we used $\phi_{R}=\phi_{R}^{i}+\phi_{R}^{b m}+\phi_{R}^{b g}$. It follows that $1 / f_{b 0}=1+\phi_{R}^{i} / \phi_{R}^{b m}$ and thus that in this ansatz the ratio between inactive and ribosomal mass fraction remains constant.

\section{Data sets}

\section{Growth rate and protein mass fraction}

We used data from Metzl-Raz et al. (2017) (S. cerevisiae), Dai et al. (2016) (E. coli), Fraenkel and Neidhardt (1961) (A. aerogenes), Alberghina et al. (1975) (N. crassa), Brown and Rose (1969) (C. utilis), Cook (1963) (E. gracilis) in Figures 3, 1 - Supplement 1 and 2 - Supplement 3.

\section{Experimental data}

We compiled two data sets from the literature relative to degradation rates in E. coli and S. cerevisiae. These data are available as a Mendeley Data repository at the following address http://dx.doi.org/10.17632/85pxpdsx38.1.

For E. coli, we considered data of the average protein degradation rate from Pine (1970); Nath and Koch (1970); Pine (1973); Mosteller et al. (1980); Larrabee et al. (1980); J M Gancedo (1982). For S. cerevisiae, we considered data from J M Gancedo (1982); Helbig et al. (2011); Christiano et al. (2014); Martin-Perez and Villén (2017). These studies can be divided into two categories according to their experimental design:

1. studies that provide a distribution of degradation rates by measuring the half-life of hundreds or thousands of proteins. Out of these studies, we estimated the mean degradation rate as the average of this distribution. In E. coli, Mosteller et al. (1980); Larrabee et al. (1980) provide a distribution of degradation rates by combining pulse-chase experiments with 2 -D gel electrophoresis. We note that these authors measure $\approx 100$ degradation rates, but there are more than 4000 E. coli proteins. In S. cerevisiae, Helbig et al. (2011); Christiano et al. (2014); Martin-Perez and Villén (2017) measure the half-lives of thousands of protein by combining metabolic labelling and mass spectrometry. Christiano et al. (2014); Martin-Perez and Villén (2017) perform SILAC experiments, which are based on amino acid labelling, while Helbig et al. (2011) uses stable heavy nitrogen isotopes for labelling.

2. studies that measure total protein content breakdown and use data analysis to infer the mean degradation rate. All such studies never measure directly the degradation dynamics of specific proteins, but only the dynamics of total protein content. In E. coli, Pine (1970); Nath and Koch (1970); Pine (1973) provide a single mean degradation rate. Nath and Koch (1970) also attempts to estimate the rate of two distinct protein classes, respectively fast-degrading and slow-degrading types. In S. cerevisiae, J M Gancedo (1982) uses the same type of set-up. All these studies perform pulse-chase experiments by labelling completely the proteome of the cell by incorporation of radioactively-labelled amino acids. After switching to incorporation of unlabelled amino acids, the total amount of labelled protein can either stay constant or decrease due to degradation. For all these studies, we performed our own data analysis on the the provided raw data and estimated the mean degradation rate from the rate of decrease of the labelled total cell protein. We describe below the methods of our data analysis. 


\section{Data analysis}

We begin this section by considering the work of Pine (1973), our main source in the main text for degradation rates across growth conditions. In this case, we have followed the author's estimates since the raw data are provided only for few conditions, but we have re-examined critically their assumption. The authors estimate the mean degradation rate by assuming that the labelled cell protein decreases with a single degradation rate. Mathematically, this means that

$$
P_{L}(t)=P_{L}^{0} \exp (-\eta t),
$$

with $P_{L}(t)$ being the amount of labelled protein at time $t$ after the pulse period. This allows to estimate $\eta$ as

$$
\eta=-\frac{1}{t} \log \left(\frac{P_{L}(t)}{P_{L}^{0}}\right),
$$

or any equivalent combination. We note that this method provides a good estimate even if the degradation rate differs from protein to protein. To see this, we re-write equation (22):

$$
P_{L}(t)=\sum_{i} P_{L i}^{0} \exp \left(-\eta_{i} t\right)
$$

where the sum runs over all the proteins in the cell. By considering the initial fraction of proteins having degradation rate $\eta$, we can write this in terms of the distribution $P(\eta)$.

$$
\log \left(\frac{P_{L}(t)}{P_{L}^{0}}\right)=\int P(\eta) \exp (-\eta t) d \eta=\langle\exp (-\eta t)\rangle,
$$

where the sign $\langle\cdot\rangle$ indicates performing an average.

Since approximately

$$
\langle\exp (-\eta t)\rangle \approx \exp (-\langle\eta\rangle t),
$$

the previous equation still holds in the mean,

$$
\langle\eta\rangle \approx-\log \left(\frac{P_{L}(t)}{P_{L}^{0}}\right) \frac{1}{t} .
$$

Jensen's inequality implies that this estimate always underestimates the true mean degradation rate, hence, the experimental data points shown in Fig. 3 could be considered as lower bounds.

For Nath and Koch (1970); Pine (1970), we estimated the mean degradation by the dividing the cell protein content in three classes, one of which consists of stable proteins. The other two classes represent respectively fast and slow degrading proteins. This approach is directly inspired by the ideas of Nath and Koch (1970).

The total protein content will decay in general according to the following equation:

$$
P_{L}(t)=P_{\text {fast }}^{0} \exp \left(-\eta_{\text {fast }} t\right)+P_{\text {slow }}^{0} \exp \left(-\eta_{\text {slow }} t\right)+P_{\text {stable }}^{0}
$$

or as a fraction of initial amount of labelled protein

$$
\frac{P_{L}(t)}{P_{L}^{0}}=f_{\text {fast }} \exp \left(-\eta_{\text {fast }} t\right)+f_{\text {slow }} \exp \left(-\eta_{\text {slow }} t\right)+f_{\text {stable }}
$$

with $f_{\text {fast }}, f_{\text {slow }}$ and $f_{\text {stable }}$ being the probability that a protein belongs to one of the three classes.

The mean degradation rate will be: 


$$
\langle\eta\rangle=f_{\text {fast }} \eta_{\text {fast }}+f_{\text {slow }} \eta_{\text {slow }}
$$

To estimate this, we must infer the parameters $f_{\text {fast }}, \eta_{\text {fast }}, f_{\text {slow }}$ and $\eta_{\text {slow }}$ from equation (29). In practice, we are able to reduce the number of parameters on a case-by-case basis.

Nath and Koch (1970) and J M Gancedo (1982) perform this analysis themselves, and assume that the slow class is indeed slow enough to approximate the exponential to a linear function. They derive equation $(29)$ and obtain:

$$
-\frac{1}{P_{L}^{0}} \frac{d P_{L}(t)}{d t}=f_{\text {fast }} \eta_{\text {fast }} \exp \left(-\eta_{\text {fast }} t\right)+f_{\text {slow }} \eta_{\text {slow }}
$$

They fit $f_{\text {fast }}, \eta_{\text {fast }}$ and $f_{\text {slow }} \eta_{\text {slow }}$ to the experimental curve. We are able to extract the mean degradation rate out of these parameters.

Pine (1970) do not perform this analysis. By performing it ourselves, we find that using only two classes fits the data well using the following expression:

$$
\frac{P_{L}(t)}{P_{L}^{0}}=f_{\text {fast }} \exp \left(-\eta_{\text {fast }} t\right)+\left(1-f_{\text {fast }}\right)
$$

We extract $f_{\text {fast }}$ and $\eta_{\text {fast }}$ from the fit and use it to compute the mean degradation rate.

\section{Data interpolation and extrapolation}

Many estimates and calculations in the main text require the combined knowledge of ribosomal protein fractions, degradation rates and translation elongation rates, all the same growth rate. We obtained all these observables from different sources, and unfortunately combined measurements from the same dataset are almost never available.

In order to use different measurements in our calculations, we interpolated the data in different ways. In the following, we list all the operations that we performed on the data for this purpose:

1. in Figure 3 and 4 , we performed a linear fit on degradation data from Pine (1973), and used the continuous interpolation of these data to obtain degradation rates at the same growth rates measured by Dai et al. (2016);

2. we performed a linear fit on J M Gancedo (1982) degradation data and used it to obtain degradation rates at the growth rates measured by Metzl-Raz et al. (2017);

3. in Figure 3, we obtained continuous curves of the active ribosomal fraction by performing a polynomial fit on the ribosomal fraction data, the translation elongation rate data by Dai et al. (2016) and the ribosomal fraction data by Metzl-Raz et al. (2017). This was done in order to avoid the effect of noisy measurements. Note that no such interpolation was done to obtain the points (crosses and circles) shown in 3

4. for S. cerevisiae, translation elongation rates measurements across growth conditions are not available. Ref. Metzl-Raz et al. (2017) argues that the elongation rate is likely constant across growth conditions. We followed this assumption and set the elongation translation equal to the inverse of the slope of the first growth law measured in Metzl-Raz et al. (2017). 
Table 1. Summary of the symbols used in the text.

\begin{tabular}{ll}
\hline Symbol & Definition \\
\hline$M$ & total protein mass \\
$M_{R}$ & total ribosomal protein mass \\
$\phi_{R}=M_{R} / M$ & mass fraction of ribosomal proteins \\
$\lambda$ & growth rate \\
$J_{\mathrm{tl}}$ & mass translational flux \\
$m_{p}$ & typical protein mass \\
$m_{R}$ & protein mass of a ribosome \\
$R_{b}$ & number of transcript-bound ribosomes \\
$R_{a}$ & number of active ribosomes \\
$R_{i}$ & number of inactive ribosomes \\
$R_{f}$ & number of free ribosomes, available to bind the mRNA \\
$R=R_{i}+R_{f}+R_{b}$ & total number of ribosomes in our framework \\
$R=R_{i}+R_{a}$ & total number of ribosomes in the standard framework \\
$k$ & codon elongation rate \\
$L_{R}$ & total number of aa in a ribosome \\
$\gamma=k / L_{R}$ & inverse of typical time to translate all amino-acids of a ribosome \\
$\phi_{R}=M_{R} / M=m_{R} R / M$ & protein mass fraction of ribosomal proteins \\
\hline
\end{tabular}

\section{Acknowledgments}

MCL and LCa are funded by the Italian Association for Cancer Research AIRC-IG (REF: 23258), and LCa by was funded by the AIRC Fellowship (REF: 23870).

MO is supported by the Departments of Excellence 2018-2022 Grant awarded by the Italian Ministry of Education, University and Research (MIUR) (Grant No. L. 232/2016)

\section{References}

Alberghina, F., Sturani, E., and Gohlke, J. (1975). Levels and rates of synthesis of ribosomal ribonucleic acid, transfer ribonucleic acid, and protein in neurospora crassa in different steady states of growth. Journal of Biological Chemistry, 250(12):261-272.

Beese, C. J., Brynjólfsdóttir, S. H., and Frankel, L. B. (2020). Selective Autophagy of the Protein Homeostasis Machinery: Ribophagy, Proteaphagy and ER-Phagy. Frontiers in Cell and Developmental Biology, 7(January):1-12.

Belle, A., Tanay, A., Bitincka, L., Shamir, R., and O'Shea, E. K. (2006). Quantification of protein half-lives in the budding yeast proteome. Proceedings of the National Academy of Sciences, 103(35):13004-13009.

Biselli, E., Schink, S. J., and Gerland, U. (2020). Slower growth of escherichia coli leads to longer survival in carbon starvation due to a decrease in the maintenance rate. Molecular systems biology, 16:e9478.

Boehlke, K. W. and Friesen, J. D. (1975). Cellular content of ribonucleic acid and protein in saccharomyces cerevisiae as a function of exponential growth rate: calculation of the apparent peptide chain elongation rate. Journal of Bacteriology, 121(2):429-433.

Borkowski, O., Ceroni, F., Stan, G. B., and Ellis, T. (2016). Overloaded and stressed: whole-cell considerations for bacterial synthetic biology. Current Opinion in Microbiology, 33:123-130. 
Bosdriesz, E., Molenaar, D., Teusink, B., and Bruggeman, F. J. (2015). How fastgrowing bacteria robustly tune their ribosome concentration to approximate growth-rate maximization. The FEBS Journal, 282(10):2029-2044.

Brown, C. M. and Rose, A. H. (1969). Effects of temperature on composition and cell volume of candida utilis. Journal of Bacteriology, 97(1):261-272.

Christiano, R., Nagaraj, N., Fröhlich, F., and Walther, T. C. (2014). Global proteome turnover analyses of the yeasts s. cerevisiae and s. pombe. Cell Reports, 9(5):1959-1965.

Ciandrini, L., Stansfield, I., and Romano, M. C. (2013). Ribosome traffic on mrnas maps to gene ontology: Genome-wide quantification of translation initiation rates and polysome size regulation. PLoS Comput. Biol., 9.

Cook, J. R. (1963). Adaptations in growth and division in euglena effected by energy supply*. The Journal of Protozoology, 10(4):436-444.

Dai, X. and Zhu, M. (2020). Coupling of ribosome synthesis and translational capacity with cell growth. Trends in biochemical sciences, 45:681-692.

Dai, X., Zhu, M., Warren, M., Balakrishnan, R., Patsalo, V., Okano, H., Williamson, J. R., Fredrick, K., Wang, Y.-P., and Hwa, T. (2016). Reduction of translating ribosomes enables escherichia coli to maintain elongation rates during slow growth. Nature microbiology, 2:16231.

DeLong, J. P., Okie, J. G., Moses, M. E., Sibly, R. M., and Brown, J. H. (2010). Shifts in metabolic scaling, production, and efficiency across major evolutionary transitions of life. Proceedings of the National Academy of Sciences, 107(29):12941-12945.

Fraenkel, D. G. and Neidhardt, F. C. (1961). Use of chloramphenicol to study control of rna synthesis in bacteria. Biochimica et Biophysica Acta, 53(1):96 - 110.

Gefen, O., Fridman, O., Ronin, I., and Balaban, N. Q. (2014). Direct observation of single stationary-phase bacteria reveals a surprisingly long period of constant protein production activity. Proceedings of the National Academy of Sciences, 111(1):556-561.

Goldberg, A. and Dice, J. (1974). Intracellular protein degradation in mammalian and bacterial cells. Annual Review of Biochemistry, 43(1):835-869. PMID: 4604628.

Gray, D. A., Dugar, G., Gamba, P., Strahl, H., Jonker, M. J., and Hamoen, L. W. (2019). Extreme slow growth as alternative strategy to survive deep starvation in bacteria. Nature Communications, 10.

Greulich, P., Ciandrini, L., Allen, R. J., and Romano, M. C. (2012). Mixed population of competing totally asymmetric simple exclusion processes with a shared reservoir of particles. Phys. Rev. E, 85.

Helbig, A. O., Daran-Lapujade, P., van Maris, A. J. A., de Hulster, E. A. F., de Ridder, D., Pronk, J. T., Heck, A. J. R., and Slijper, M. (2011). The diversity of protein turnover and abundance under nitrogen-limited steady-state conditions in saccharomyces cerevisiae. Mol. BioSyst., 7:3316-3326.

J M Gancedo, S López, F. B. (1982). Calculation of half-lives of proteins in vivo. heterogeneity in the rate of degradation of yeast proteins. Molecular and Cellular Biochemistry, 43(89-95):436-444. 
Kafri, M., Metzl-Raz, E., Jonas, F., and Barkai, N. (2016). Rethinking cell growth models. FEMS yeast research, 16.

Kempes, C. P., van Bodegom, P. M., Wolpert, D., Libby, E., Amend, J., and Hoehler, T. (2017). Drivers of bacterial maintenance and minimal energy requirements. Frontiers in microbiology, 8:31.

Kempes, C. P., Wang, L., Amend, J. P., Doyle, J., and Hoehler, T. (2016). Evolutionary tradeoffs in cellular composition across diverse bacteria. The ISME journal, 10:21452157.

Klumpp, S., Scott, M., Pedersen, S., and Hwa, T. (2013). Molecular crowding limits translation and cell growth. Proceedings of the National Academy of Sciences of the United States of America, 110:16754-16759.

Larrabee, K. L., Phillips, J. O., Williams, G. J., and Larrabee, A. R. (1980). The relative rates of protein synthesis and degradation in a growing culture of escherichia coli. Journal of Biological Chemistry, 255(9):4125-30.

Li, G. W. (2015). How do bacteria tune translation efficiency? Current Opinion in Microbiology, 24:66-71.

Long, A. M., Hou, S., Ignacio-Espinoza, J. C., and Fuhrman, J. A. (2021). Benchmarking microbial growth rate predictions from metagenomes. The ISME journal, 15(1):183-195.

Maitra, A. and Dill, K. A. (2015). Bacterial growth laws reflect the evolutionary importance of energy efficiency. Proceedings of the National Academy of Sciences of the United States of America, 112:406-411.

Mandelstam, J. (1958). Turnover of protein in growing and non-growing populations of escherichia coli. The Biochemical journal, 69(1):110-119.

Martin-Perez, M. and Villén, J. (2017). Determinants and regulation of protein turnover in yeast. Cell Systems, 5(3):283-294.e5.

Maurizi, M. (1992). Proteases and protein degradation in escherichia coli. Experientia, 48(2):178-201.

Metzl-Raz, E., Kafri, M., Yaakov, G., Soifer, I., Gurvich, Y., and Barkai, N. (2017). Principles of cellular resource allocation revealed by condition-dependent proteome profiling. eLife, 6 .

Mosteller, R. D., Goldstein, R. V., and Nishimoto, K. R. (1980). Metabolism of individual proteins in exponentially growing escherichia coli. The Journal of biological chemistry, 255(6):2524-2532.

Nath, K. and Koch, A. L. (1970). Protein degradation in escherichia coli: I. measurement of rapidly and slowly decaying components. Journal of Biological Chemistry, 245(11):2889-2900.

Nath, K. and Koch, A. L. (1971). Protein degradation in escherichia coli : Ii. strain differences in the degradation of protein and nucleic acid resulting from starvation. Journal of Biological Chemistry, 246(22):6956-6967.

Pine, M. J. (1970). Steady-state measurement of the turnover of amino acid in the cellular proteins of growing escherichia coli: Existence of two kinetically distinct reactions. Journal of Bacteriology, 103(1):207-215. 
Pine, M. J. (1973). Regulation of intracellular proteolysis in escherichia coli. Journal of bacteriology, 115(1):107-116.

Santra, M., Farrell, D. W., and Dill, K. A. (2017). Bacterial proteostasis balances energy and chaperone utilization efficiently. Proceedings of the National Academy of Sciences of the United States of America, 114:E2654-E2661.

Schink, S. J., Biselli, E., Ammar, C., and Gerland, U. (2019). Death rate of e. coli during starvation is set by maintenance cost and biomass recycling. Cell systems, 9:64-73.e3.

Schroer, D. W. and St. John, A. C. (1981). Relative stability of membrane proteins in escherichia coli. Journal of Bacteriology, 146(2):476-483.

Scott, M. and Hwa, T. (2011). Bacterial growth laws and their applications. Current opinion in biotechnology, 22:559-565.

Scott, M., Mateescu, E. M., Zhang, Z., and Hwa, T. (2010). Interdependence of Cell Growth Origins and Consequences. Science, 330(November):1099-1102.

Shaw, L. B., Zia, R. K., and Lee, K. H. (2003). Totally asymmetric exclusion process with extended objects: a model for protein synthesis. Phys. Rev. E.

Zhang, T., Wolfe, C., Pierle, A., Welle, K. A., Hryhorenko, J. R., and Ghaemmaghami, S. (2017). Proteome-wide modulation of degradation dynamics in response to growth arrest. Proceedings of the National Academy of Sciences of the United States of America, 114:E10329-E10338.

Zhu, M., Mu, H., Jia, M., Deng, L., and Dai, X. (2020). Control of ribosome synthesis in bacteria: the important role of rrna chain elongation rate. Science China. Life sciences. 
(a)

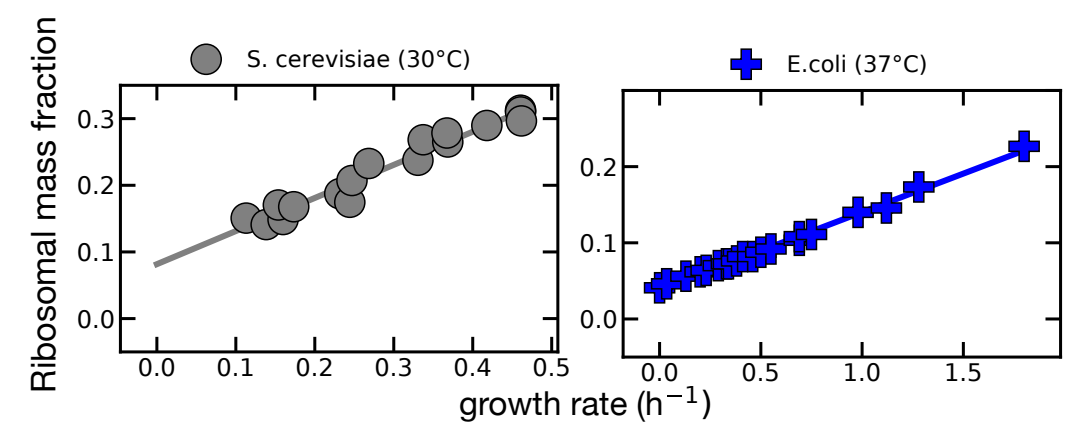

(b)

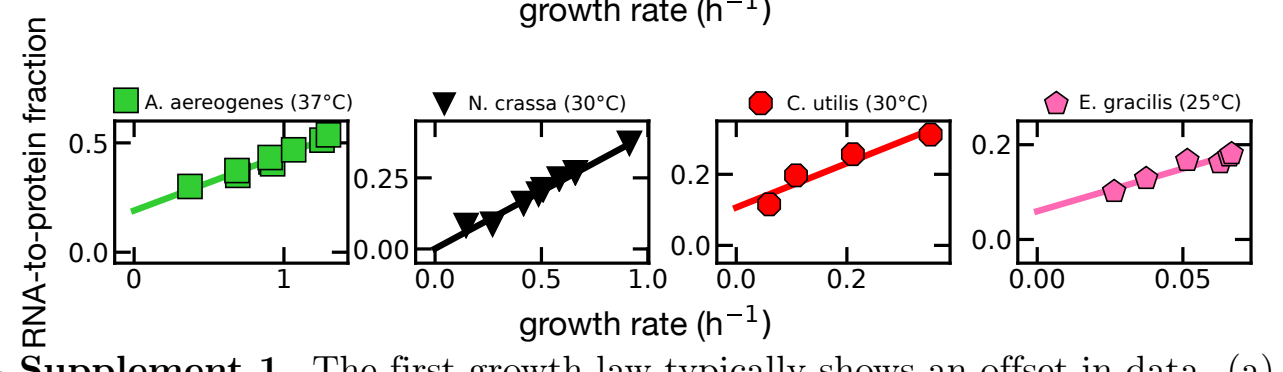

Fig 1 - Supplement 1. The first growth law typically shows an offset in data. (a) Data on ribosomal mass fraction for E. coli and S. cerevisiae. (b) Data on RNA/protein ratios for other organisms. Data from Metzl-Raz et al. (2017) (S. cerevisiae), Dai et al. (2016) (E. coli), Fraenkel and Neidhardt (1961) (A. aerogenes), Alberghina et al. (1975) (N.crassa), Brown and Rose (1969) (C. utilis), Cook (1963) (E. gracilis). 

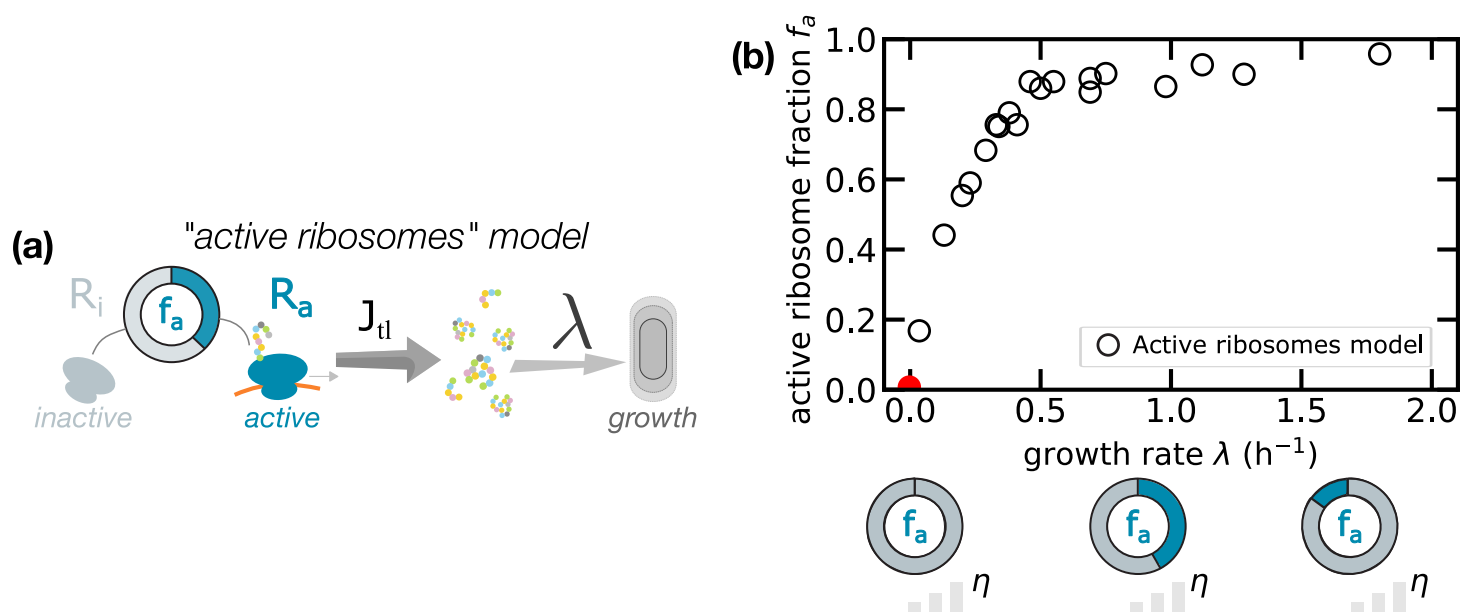

Fig 2 - Supplement 1. (a) In the standard framework ribosomes are divided in two categories - active and inactive - and only the fraction $f_{a}$ of active ribosomes is responsible for protein production. (b) The plot reports the estimated $f_{a}$ (circles) assuming this model and using data from Dai et al. (2016). The red circle represents the extrapolated point at zero growth.
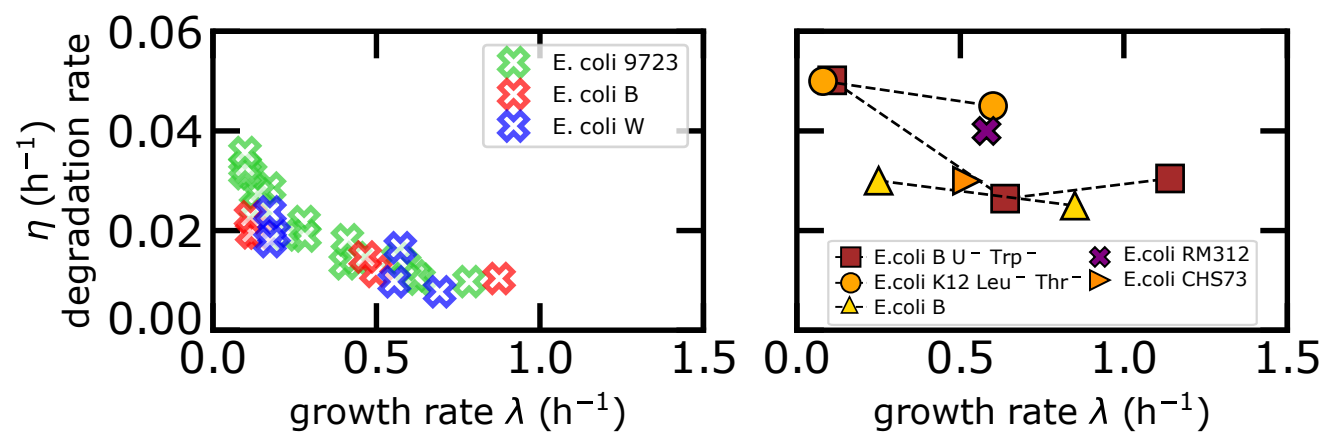

Fig 2 - Supplement 2. (a) Degradation rate across growth conditions from Pine (1973) as used in the main text. (b) Degradation rate across different growth conditions from other studies using different strains and techniques, Nath and Koch (1970) (E. coli $B U^{-1} \operatorname{Trp}^{-1}$ ), Mandelstam (1958) (E. coli K12 Leu ${ }^{-1}$ Thr $\left.^{-1}\right)$, Pine (1970) (E. coli B), Mosteller et al. (1980) (E. coli RM132), Larrabee et al. (1980) (E. coli CHS73). 

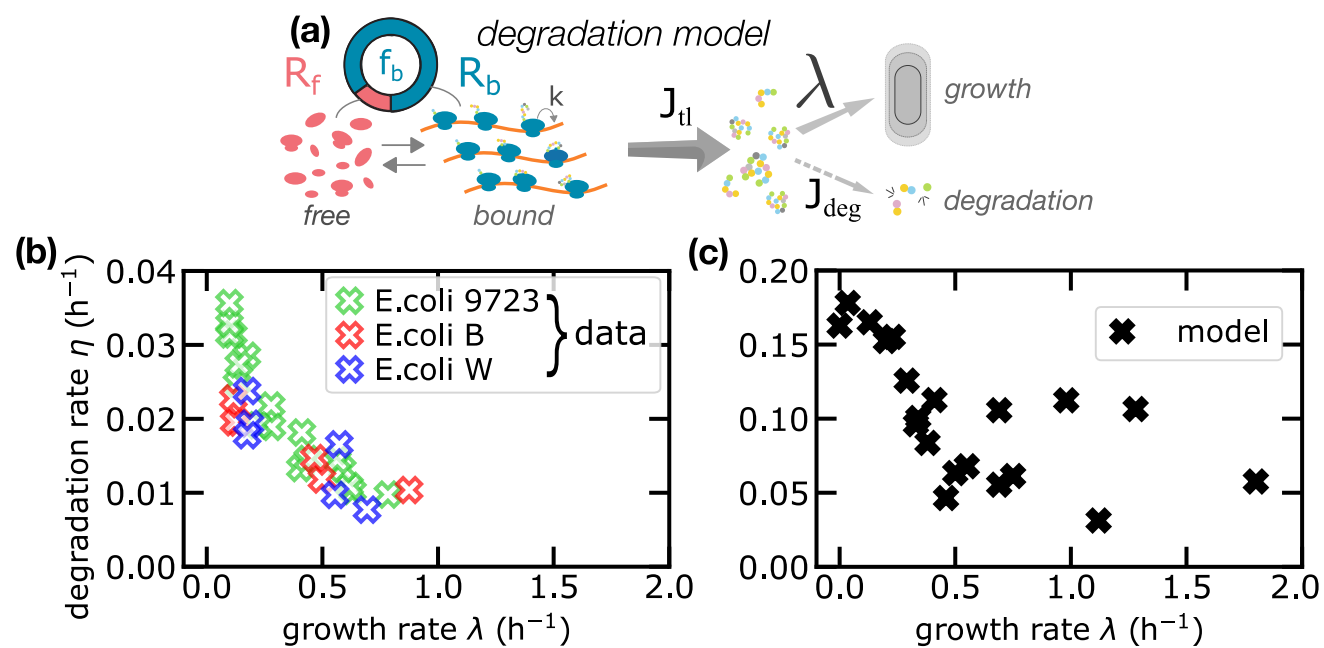

Fig 2 - Supplement 3. The model with degradation and no inactive ribosomes captures qualitatively, but not quantitatively the trend of degradation rates in E. coli. (a) Sketch of the first model of protein production proposed in this work, which includes protein degradation but no inactive ribosomes. In this model, ribosomes follow a first-order kinetics to bind the transcripts, and all bound ribosomes contribute to protein synthesis (mass production). (b) Degradation rate across growth conditions from Pine (1973). (c) Degradation rate estimated from Dai et al. (2016) using the model in the first panel. The model captures the qualitative trend of the degradation rate across growth conditions, but fails quantitatively by overestimating the rates by a factor of 4 . 

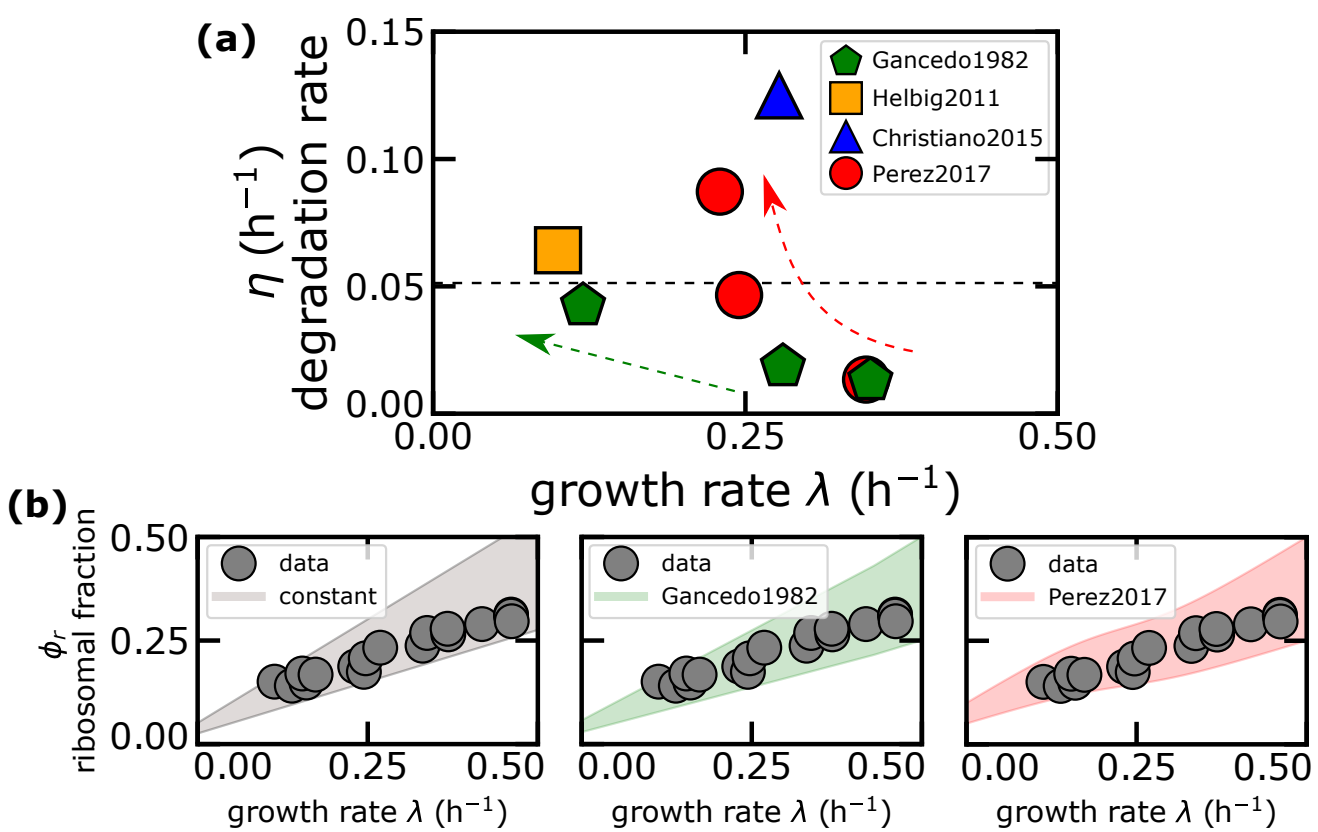

Fig 2 - Supplement 4. The degradation-only model is compatible with data for $S$. cerevisiae, given the uncertainty in the parameters. (a) Mean degradation rate across growth conditions from J M Gancedo (1982); Helbig et al. (2011); Martin-Perez and Villén (2017); Christiano et al. (2014) respectively using strains CJM13, CEN.PK113-7D DBY10144 and BY4742. The dashed line indicates the average of all the shown points (which are averages in a single condition). The dashed arrow lines highlight the increasing trends of degradation rates with decreasing growth rates in two data sets. (b) The range of predicted ribosomal fractions of the model, plotted next to data points from Metzl-Raz et al. (2017) that uses strain BY4742. The model requires as inputs degradation rates and translation elongation rates. As a value for the degradation rate, we have taken the mean value, shown in panel a as a dashed line in the left subpanel, as well as a linear fit of the degradation rate from J M Gancedo (1982) (green hexagons in panel a, central subpanel in panel b), and a sigmoid fit for Martin-Perez and Villén (2017) (red circles in panel a, right-hand subpanel in panel b), see also Methods and Materials. We then considered a range of physiologically relevant translation elongation rates $\left(3-8\right.$ aa s $\left.^{-1}\right)$ from ref. (Boehlke and Friesen, 1975). The shaded area represents the prediction of the model for such range.

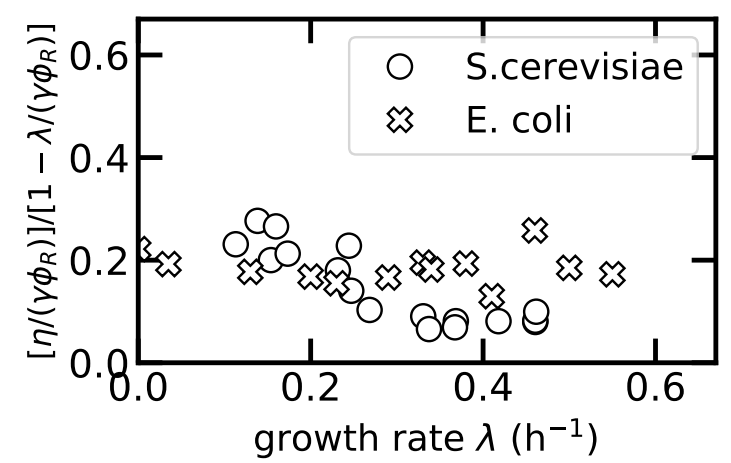

Fig 3 - Supplement 1. Experimental support of the constant-ratio ansatz -Eq. (15) in the main text. The plot shows that the ratio $\left[\eta /\left(\gamma \Phi_{R}\right)\right] /\left[1-\lambda /\left(\gamma \phi_{R}\right)\right]$, evaluated from the available E. coli and S. cerevisiae data (see Methods and Materials), is compatible with a constant $f_{b 0} \simeq 0.2$, across growth conditions, especially for the (much more precise) E. coli data. 


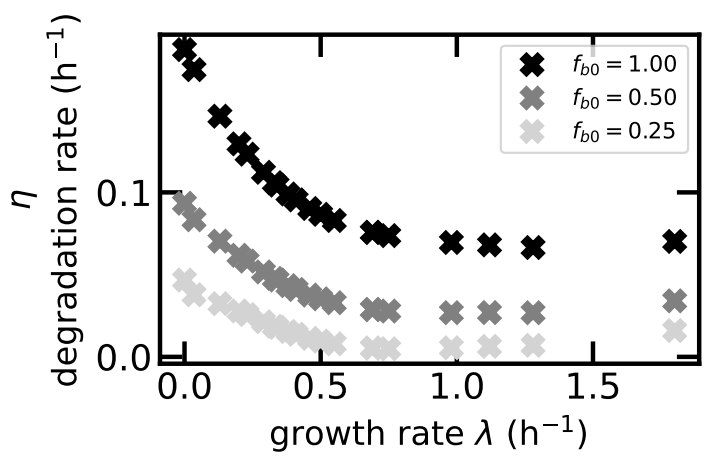

Fig 3 - Supplement 2. Mean degradation rates across growth conditions as predicted by the model with the constant-ratio ansatz for the fraction of bound/active ribosomes. The plot shows degradation rates as predicted by the model equation $\eta=f_{b} \phi_{r} \gamma-\lambda$ with $f_{b}$ equal to the constant-ratio ansatz from Eq. (14). $\phi_{R}$ and $\gamma$ are taken from E. coli data given in Dai et al. (2016). 